\title{
The "thymopericardial fat flap": A versatile flap in thoracic and cardiovascular surgery
}

\author{
Alain Wurtz, MD, ${ }^{\text {a }}$ Francis Juthier, MD, PhD, ${ }^{\mathrm{b}}$ Massimo Conti, MD, ${ }^{\mathrm{a}}$ and André Vincentelli, MD, PhD, ${ }^{\mathrm{b}}$ \\ Lille, France
}

Supplemental material is available online.

Major tracheal reconstructive surgery requires a flap to reinforce the suture line and prevent erosion of contiguous large vessels. Omentoplasty, myoplasty, or flaps with a blood supply arising from the branches of the internal thoracic (IT) artery such as the "pericardial fat graft" "thymus flap", can be used. We describe the "thymopericardial fat flap" (TPF), which includes the IT pedicle, overlying mediastinal pleura, homolateral thymic lobe, and continuous pericardial fat tissue.

In adulthood, the intrathoracic portion of the thymic lobes remains as fat and connective tissue lying on the anterosuperior pericardium. Usually, one major thymic branch per lobe comes from the IT artery. ${ }^{3}$ The pericardial fat tissue extends up to the caudal portion of the thymus and down the cardiophrenic angles, with the blood supply arising from the IT arteries (Figure 1).

During major tracheal reconstructive surgery, the right TPF is mobilized through a median sternotomy combined with a collar incision. Given the length, IT pedicle harvesting is mandatory to ensure the viability of the distal part of flap (pericardial fat tissue). The thyroid isthmus and both thymic lobes are divided medially, with preservation of the superior thymic veins. To expose the undersurface of the right half of the sternum, an externally positioned sternal retractor is used. The initial part of the IT pedicle, with its adjacent fat and parietal pleura, is dissected up to the posterior face of the first costal cartilage, with preservation of the internal thoracic vein at its junction with the innominate vein. The incision is extended along the IT pedicle, down to the sixth intercostal space. The pedicle is freed from the thoracic wall caudally. The intercostal arteries and accompanying veins are identified and divided be-

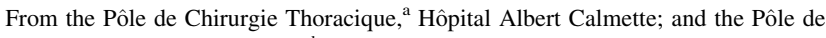
Chirurgie Cardio-Vasculaire, ${ }^{\mathrm{b}}$ Hôpital Cardiologique, CHU Lille, Lille, France. Disclosures: Authors have nothing to disclose with regard to commercial support. Received for publication Jan 19, 2010; revisions received June 23, 2010; accepted for publication July 6, 2010; available ahead of print Aug 23, 2010.

Address for reprints: Alain Wurtz, MD, Pôle de Chirurgie Thoracique, Hôpital Albert Calmette, CHU Lille, F59037 Lille Cedex France (E-mail: alain.wurtz@ chru-lille.fr).

J Thorac Cardiovasc Surg 2011;141:841-2

$0022-5223 / \$ 36.00$

Copyright (c) 2011 by The American Association for Thoracic Surgery doi:10.1016/j.jtcvs.2010.07.005
}

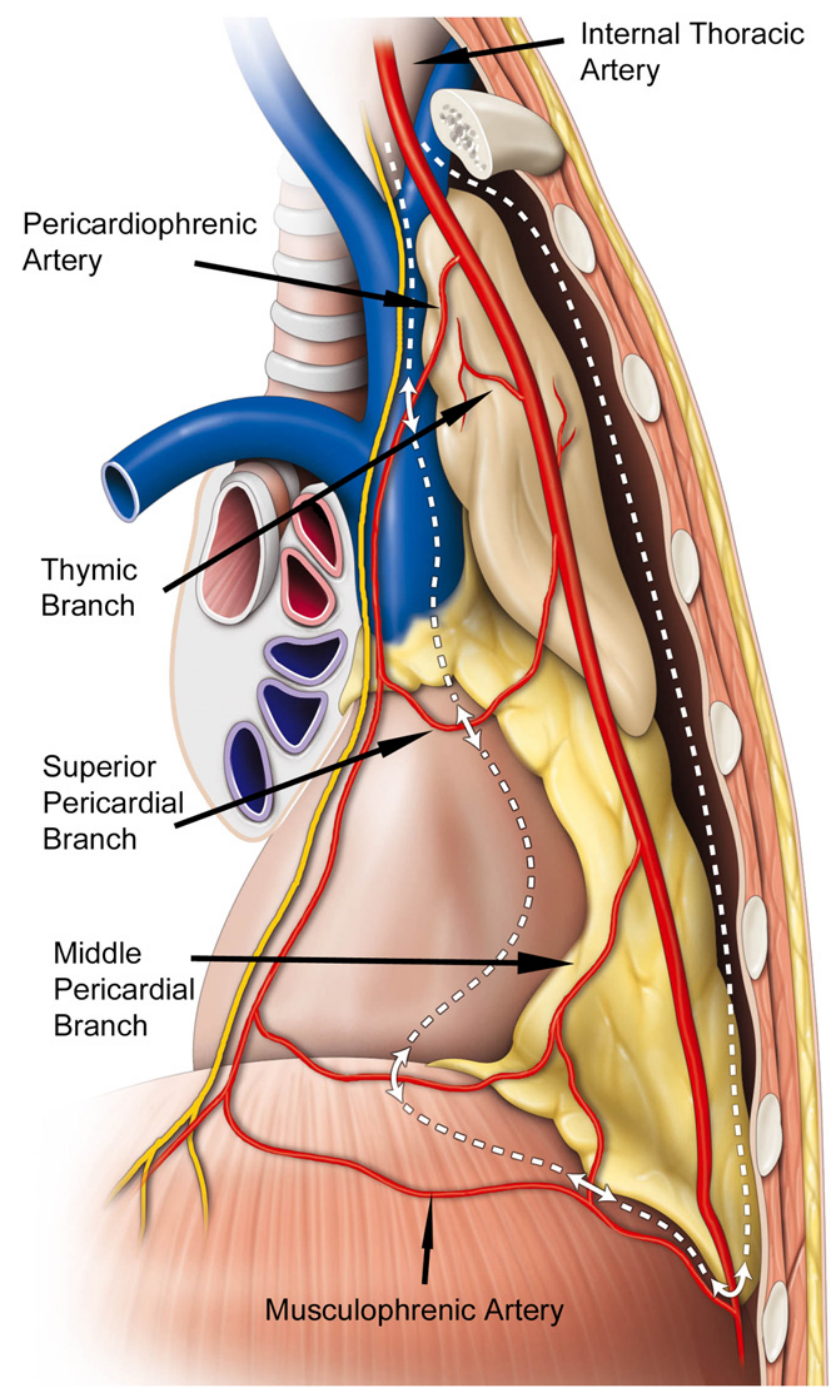

FIGURE 1. Anatomy and development of "thymopericardial fat flap." Diagram showing "thymopericardial fat flap" fashioned using internal thoracic artery (and vein), freeing from thoracic wall, divided in sixth intercostal space, and divided from pericardiophrenic and musculophrenic arteries (double arrows and dotted line).

tween surgical clips. The IT pedicle is divided in the sixth intercostal space. The flap is then freed from the ascending aorta and pericardium. Finally, the anastomotic vessels from the pericardiophrenic and musculophrenic branches are divided using a transpleural approach (Figure 1), allowing "en bloc" mobilization of an approximately 20 -cm-long $\times 5$-cm-wide well-vascularized flap (Figure E1). 


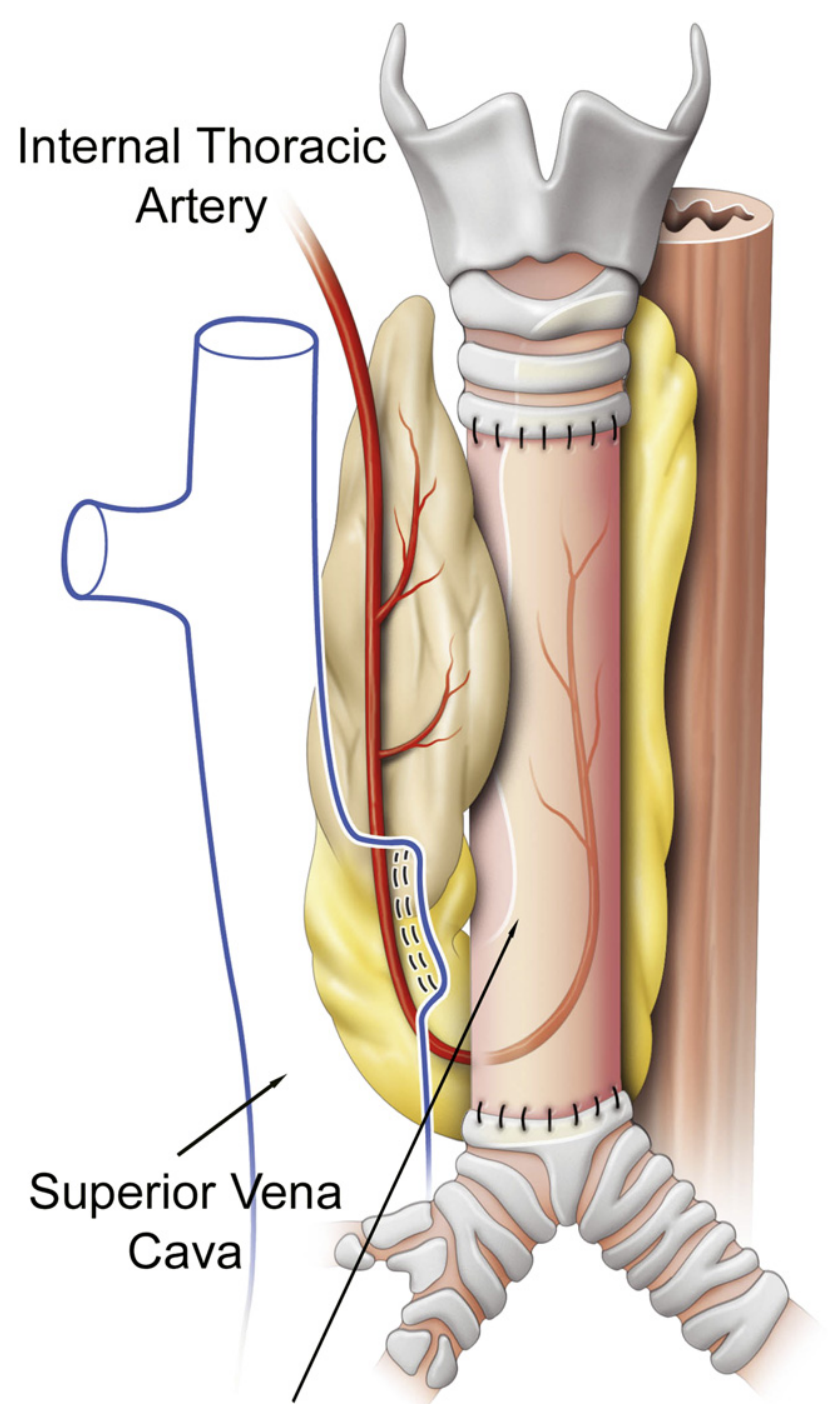

\section{Aortic Allograft}

FIGURE 2. "Thymopericardial fat flap" mobilized as interposition between splinted aortic allograft, superior vena cava, and esophagus in setting of tracheal replacement.

\section{RESULTS}

The TPF was used in 4 men and 1 woman aged 17 to 56 years who had large adenoid cystic carcinoma $(n=2)$, postintubation stenosis $(n=2)$, or carcinoid tumor $(n=1)$. The first 2 patients underwent tracheal replacement. The TPF was mobilized to create an interposition between the aortic allograft and esophagus, down the posterior face of the carina up to the posterior face of the upper trachea (Figure 2), and the wrap was achieved using a pectoral muscle flap interposed between the allograft and large vessels. ${ }^{4}$ The last 3 patients underwent 3- to $4.5-\mathrm{cm}$-long intrathoracic tracheal resection reconstructed by primary anastomosis. The TPF was used to seal the suture line and create an interposition between the trachea and innominate artery. The mean TPF harvesting time was 56 minutes.

All but 1 patient (who experienced a sternal dehiscence) had an uneventful postoperative course. Primary anastomotic healing was achieved in all patients, and the postoperative chest computed tomography scan showed a well-vascularized TPF with a patent IT artery and vein (Figure E2). During a mean follow-up of 26 months, we did not observe any anastomotic dehiscence or fistula, and, in the 2 patients who had undergone tracheal replacement, the allograft transformed into a well-vascularized conduit. ${ }^{4}$

\section{DISCUSSION}

During tracheal replacement, an efficient allograft wrap has been shown to be mandatory to avoid life-threatening complications such as arterial erosion and esophageal fistula ${ }^{4}$ and to promote angiogenesis within the allograft. The role of flap vascularization in this process remains to be demonstrated, because studies of tracheal replacement in animal models have differed from the surgical procedure in patients by the absence of resection of the surrounding tissues facilitating angiogenesis. However, Teramachi and colleagues ${ }^{5}$ demonstrated the efficiency of the omental wrap for revascularization within a collagen-conjugated tracheal prosthesis, and we speculated that the TPF, as a "thoracic omentum," would promote angiogenesis within the aortic allograft.

Additionally, TPF is a suitable option when coverage of the suture line is mandatory after intrathoracic tracheal resection reconstructed by primary anastomosis. Furthermore, when harvested through a thoracotomy, the TPF could be used to reinforce the bronchial stump after major lung resection or to seal the suture line after a bronchoplastic procedure.

In cardiac surgery, the TPF could be considered in the field of severe aortic root endocarditis. This flap might be useful to optimize the healing process after a Bentall operation as a wrap around the prosthetic graft to protect it from infection. Furthermore, the TPF might be considered in the challenging case of prosthetic graft infection of the thoracic aorta or aortoesophageal fistula.

We are grateful to E. Kipnis for revision of the manuscript.

\section{References}

1. Brewer LA, Bai AF. Surgery of the bronchi and trachea: experience with the pedicled pericardial fat graft reinforcement. Am J Surg. 1955;89:331-46.

2. Anderson TM, Miller JI. Surgical technique and application of pericardial fat pad and pericardiophrenic grafts. Ann Thorac Surg. 1995;59:1590-1.

3. Lo Cicero J III, Michaelis LL. Interposition of the thymus as a pedicled flap in tracheal reconstructive surgery. J Trauma. 1990;30:741-4.

4. Wurtz A, Porte H, Conti M, Dusson C, Desbordes J, Copin MC, et al. Surgical technique and results of tracheal and carinal replacement with aortic allografts for salivary gland-type carcinoma. J Thorac Cardiovasc Surg. 2010;140:387-93.

5. Teramachi M, Okumura N, Nakamura T, Yamamoto Y, Kiyotani T, Takimoto Y, et al. Intrathoracic tracheal reconstruction with a collagen-conjugated prosthesis: evaluation of the efficacy of omental wrapping. J Thorac Cardiovasc Surg. 1997; 113:701-11. 


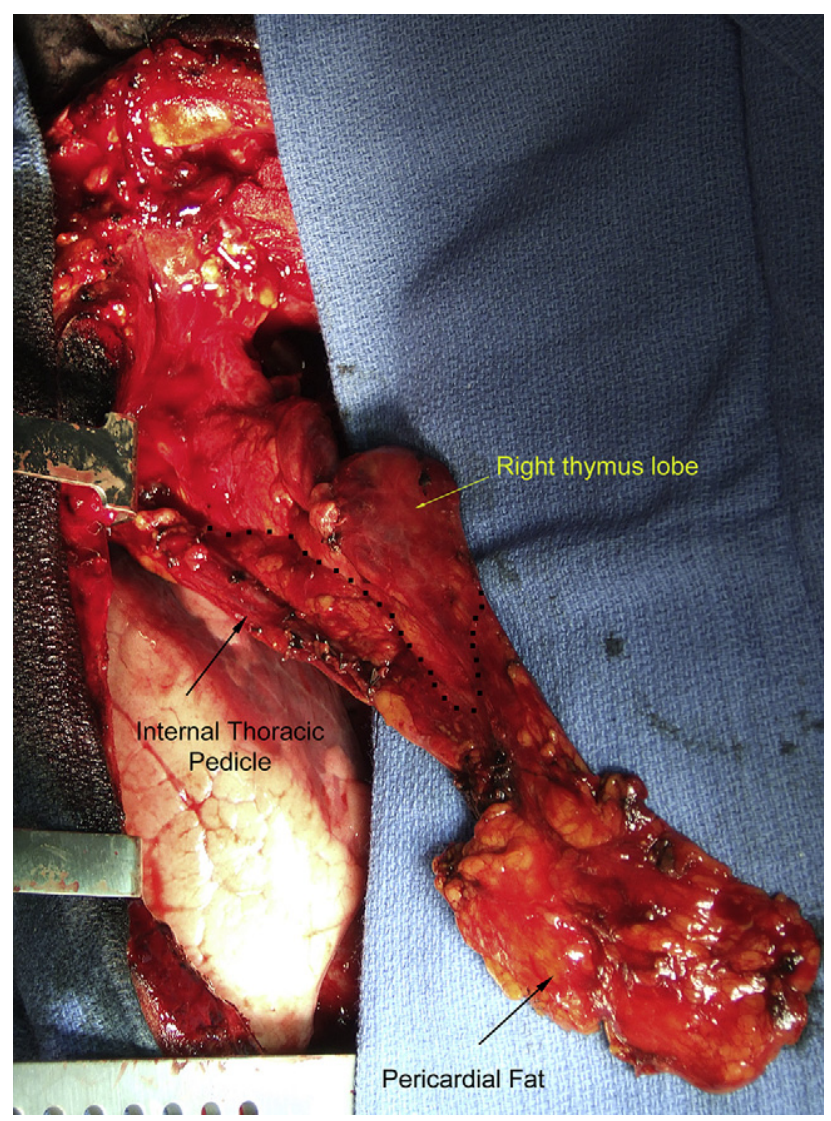

FIGURE E1. Operative view of "thymopericardial fat flap" mobilized through sternotomy in setting of tracheal replacement. Dotted line delineates thymic lobe and pericardial fat tissue.

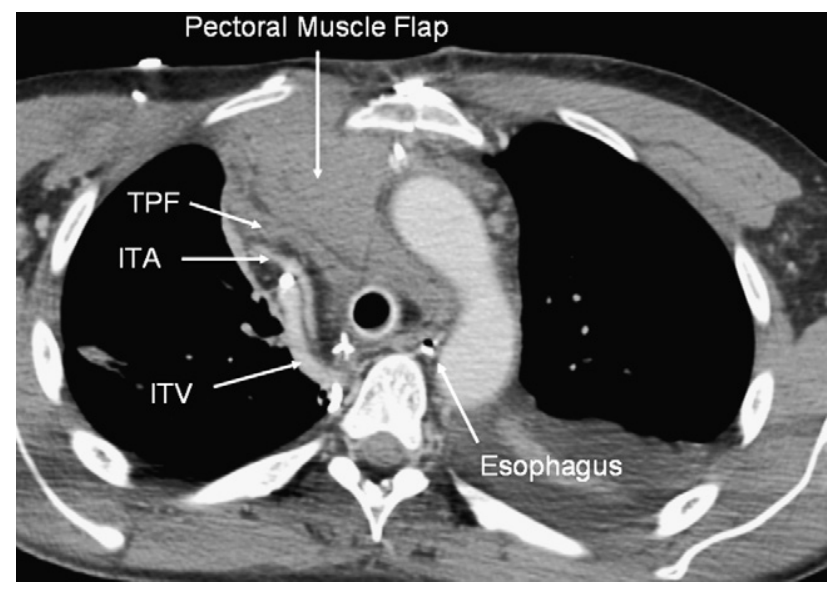

FIGURE E2. Postoperative chest computed tomography scan after tracheal replacement showing complete wrap around aortic allograft, including pectoral muscle flap and "thymopericardial fat flap" (TPF). Bolus injection of contrast medium demonstrated patent internal thoracic artery (ITA) and vein (ITV). 